Background Systemic lupus erythematosus (SLE) is characterized by the presence of autoantibodies and multi-system immune-mediated pathology. Genome-wide association studies have identified $>60$ SLE risk loci, suggesting a polygenic susceptibility. Although these loci account for significant genetic heritability, a large proportion is still missing. The missing heritability can be explained by the genetic component of intermediate phenotypes contributed by low frequency functional variants not captured on classical SNP arrays. Deep targeted sequencing of SLE associated genes allows comphensive and personalized assessment of genetic risk by annotating all common and rare disease causing variants. This study was performed to investigate functional variants in the gene for Death associated protein 1 (DAP1) that was previously implicated in susceptibility to SLE.

Methods We performed deep targeted sequencing of the DAP1 locus in 1221 SLE and 814 healthy control samples capturing both common and rare SLE associated variants. Genetic association analysis was carried out to identify disease associated haplotypes. SLE associated variants were annotated for functional efffects using publically available resources and an eQTL panel of healthy donors. Serum autoantibody signatures and gene expression profiles of SLE patients carrying SLE risk or protective geneotypes were analyzed in combination with the level of DAP1 transcription and translation. Since DAP1 protein is a potent negative regulator of autophagy, the effect of its downregulation in the risk group, was assessed in a functional autophagy assay.

Results Sequencing of the DAP1 gene revealed a novel, functional haplotype that poses risk $[\mathrm{OR}=1.5, \mathrm{p}=4.5 \mathrm{E}-$ 05] for SLE. The association was replicated in two independent cohorts of patients from different ethnic groups. RNA sequencing analysis revealed multiple cis-eQTLs embedded in the risk haplotype that downregulate DAP1 expression in immune cells. Decreased DAP1 transcription in the risk allele was consistent with reduced protein level. Healthy donors with the DAP1 risk genotype had a significantly elevated ratio of LC3-II/LC3-I in PBMCs and monocytes under starvation, suggesting enhanced autophagy mediated by the risk haplotype. SLEs with the risk genotype exhibhited significantly high autoantibody titers and altered expression of autophagy and apoptosis pathway molecules.

Conclusions This study reports a regulatory haplotype in the DAP1 locus associated with a reduced DAP1 protein level and enhanced autophagy in immune cells that can promote survival of autoreactive lymphocytes and potentiate autoimmunity.

Funding Source(s): Alliance for lupus research, NIH, UTSW THE DEVELOPMENT OF LUPUS

${ }^{1} J o a n n$ B Sweasy*, ${ }^{2}$ Rithy Meas, ${ }^{3}$ Joanne Nititham, ${ }^{3}$ Kimberly Taylor, ${ }^{4}$ Lindsey Criswell. ${ }^{1}$ Yale University School of Medicine; ${ }^{2}$ Yale University; ${ }^{3}$ Division of Rheumatology, Department of Medicine, University of California, San Francisco; ${ }^{4}$ University of California, San Francisco

\subsection{6/lupus-2019-|sm.203}

Background Recent analysis of exome chip data indicates that genetic variants (GVs) in DNA repair genes are enriched in individuals diagnosed with systemic lupus erythematosus (SLE). Methods Specifically, we analyzed 1364 single nucleotide polymorphisms (SNPs) within 41 genes that function in DNA repair. In a comparison of 1,201 SLE cases to 597 controls, corrected for ancestry, we identified $9 \mathrm{GVs}$ with odds ratios (ORs) ranging from 1.1-4.6 in genes that function in mismatch repair as being significantly enriched in SLE cases versus controls. We followed up this analysis with replication studies, by analyzing a second cohort of individuals with 1298 SLE cases and 633 controls, and we were able to replicate our initial findings with full ORs ranging from 2 to 8.393 .

Results One of the variants that emerged from our exome chip (eChip) studies is the MSH6 GV. This variant is a single amino acid change in the DNA binding domain of the MSH6 protein that is predicted to be damaging using the POLYPHEN algorithm. The MSH6 GV is eight times more prevalent in human SLE versus control cases after correcting for ancestry (odds ratio $[\mathrm{OR}]=8.393 ; \mathrm{p}=0.0398$ ). Remarkably, among the 2499 cases in the combined cohort, patients harboring the MSH6 GV are diagnosed with lupus 11.2 years earlier than SLE patients not harboring this allele $(p=0.004)$. To determine if the MSH6 GV is linked to the development of lupus, we employed CRISPr/cas9 gene-editing technology to construct a mouse harboring this variant. Initial characterization of the mice shows that they develop significantly

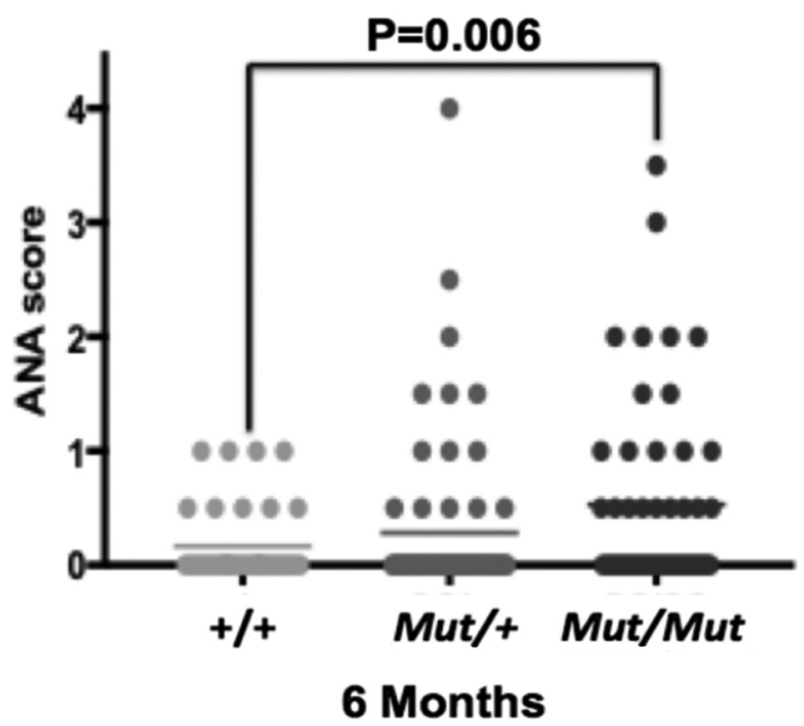

Abstract 203 Figure 1 High levels of ANA in MSH6 GV mice 
increased levels of antinuclear antibodies versus wild-type controls. Our data also suggest that the MSH6 GV identified in lupus-prone individuals results in the accumulation of mutations at $\mathrm{A}$ in the WA hotspot (W is $\mathrm{A}$ or $\mathrm{T}$; adenine or thymine, respectively; $\mathrm{A}$ is adenine) motif. Therefore, these mutations are likely to be the result of processing of the activation induced cytidine deaminase (AID)-generated $U: G$ mispair by the MSH2/6 complex followed by error-prone DNA synthesis by DNA polymerase eta.

Conclusions Importantly, these types of mutations likely result in an overall increase in positively charged amino acids in the autoantibodies that are produced, a trait that is commonly found in anti-DNA antibodies. In summary, our results suggest that the MSH6 GV has strong potential to be associated with the development of lupus.

Funding Source(s): 5 R01 ES019179-08 ; R21 AI124055-01

The MSH6GV mice develop antinuclear antibodies at 6 months of age. +/+are WT; Mut/+are heterozygotes and Mut/Mut are homozygotes.

\section{THE IMMUNE CELL LANDSCAPE IN KIDNEYS OF LUPUS NEPHRITIS PATIENTS}

${ }^{1}$ Celine C Berthier*, ${ }^{2}$ Arnon Arazi, ${ }^{3}$ Deepak Rao, ${ }^{4}$ Anne Davidson, ${ }^{5}$ Edward Browne, ${ }^{2}$ Thomas Eisenhaure, ${ }^{6}$ Nir Hacohen, ${ }^{7}$ David J Lieb, ${ }^{4}$ Betty Diamond, ${ }^{8}$ Matthias Kretzler. ${ }^{1}$ University of Michigan; ${ }^{2}$ Broad Institute; ${ }^{3}$ Brigham and Women's Hospital/Harvard Medical School; ${ }^{4}$ Feinstein Institute for Medical Research; ${ }^{5}$ UNC-Chapel Hill; ${ }^{6}$ Harvard Medical School; ${ }^{7}$ Broad Institute of MIT and Harvard; ${ }^{8}$ University of Michigan Medical School

\subsection{6/lupus-2019-Ism.204}

Background Lupus nephritis is a potentially fatal autoimmune disease, whose current treatment is ineffective and often toxic. In 2014, the National Institute of Health (NIH), industry and non-profit organizations joined their efforts with the AMP project, whose goal is to identify new diagnostic and therapeutic targets through a better understanding of the mechanisms by which individual cell types contribute to autoimmune tissue damage.

Methods To gain insights into disease mechanisms, we analyzed kidney samples from lupus nephritis patients and healthy controls using single-cell RNA-seq. Renal biopsies from 24 LN patients and 10 pre-transplant living donors (LD) were acquired across a distributed research network using a single, uniform pipeline developed by the AMP network. In brief, biopsies were cryopreserved and shipped to a centralized processing site for tissue dissociation. A total of 3541 leukocytes and 1621 epithelial cells were sorted from LN kidney samples. 438 leukocytes and 572 epithelial cells were sorted from LD biopsies. The transcriptome of those LN single tissue-infiltrating cells were assessed using single cell RNA-seq.

Results Our analysis revealed 21 subsets of leukocytes active in disease, including multiple populations of myeloid, T, NK and $\mathrm{B}$ cells, demonstrating both pro-inflammatory and resolving responses. We found evidence of local activation of B cells correlated with an age-associated B cell signature, and of progressive stages of monocyte differentiation within the kidney. A clear interferon response was observed in most cells. Two chemokine receptors, $\mathrm{C} \times \mathrm{CR} 4$ and $\mathrm{C} \times 3 \mathrm{CR} 1$, were broadly expressed, pointing to potential therapeutic targets. Gene expression of immune cells in urine and kidney was highly correlated, suggesting urine may be a surrogate for kidney biopsies.
Conclusions Our results provide a first comprehensive view of the complex network of leukocytes active in lupus nephritis kidneys. Results from this Phase 1 study identified LN active cells and pathways that can be used to guide the development of novel therapies. Analyses at a bigger scale $(n=200 \mathrm{LN})$ in Phase 2 will allow to correlate patterns and signatures of infiltrating cells with those of intrinsic renal cells, particularly the epithelial cells that make up $90 \%$ of renal cells and that are prone to hypoxic damage and cellular stress. It will accelerate the discovery of new therapeutic targets and identification of biomarkers to guide therapeutic decisions in $\mathrm{LN}$ and integrate the treatment effect.

Funding Source(s): Funding was provided through grants from the National Institutes of Health (UH2-AR067676, UH2AR067677, UH2-AR067679, UH2-AR067681, UH2-AR067685, UH2-AR067688, UH2-AR067689, UH2-AR067690, UH2AR067691, UH2-AR067694, and UM2-AR067678).

\section{SINGLE CELL RNA EXPRESSION IN LUPUS NEPHRITIS COMPARING AFRICAN-AMERICAN AND CAUCASIAN PATIENTS IDENTIFIES DIFFERENTIAL EXPRESSION OF TYPE I INTERFERON PATHWAY}

${ }^{1}$ Andrea Fava* ${ }^{2}$ Yuji Zhang, ${ }^{3}$ Nir Hacohen, ${ }^{4}$ Arnon Arazi, ${ }^{5}$ Celine C Berthier, ${ }^{6}$ Deepak Rao, ${ }^{7}$ Michael Brenner ${ }^{8}$ David Wofsy, ${ }^{9}$ Anne Davidson, ${ }^{10}$ Matthias Kretzler, ${ }^{11}$ David Hildeman, ${ }^{12}$ E Steve Woodle, ${ }^{9}$ Betty Diamond, ${ }^{13}$ Michelle Petri. ${ }^{1}$ Johns Hopkins University; ${ }^{2}$ University of Maryland; ${ }^{3}$ Harvard Medical School; ${ }^{4}$ Broad Institute; ${ }^{5}$ University of Michigan; ${ }^{6}$ Brigham and Women's Hospital/Harvard Medical School; ' Brigham And Women's Hospital; ${ }^{8}$ Russell/ Engleman Rheumatology Research Center, University of California, San Francisco, CA, USA; ${ }^{9}$ Feinstein Institute for Medical Research; ${ }^{10}$ University of Michigan Medical School; ${ }^{11}$ University of Cincinnati Department of Pediatrics; ${ }^{12}$ University of Cincinnati; ${ }^{13}$ Johns Hopkins University School of Medicine

\subsection{6/lupus-2019-Ism.205}

Background African-American ethnicity is associated with a 3fold higher risk of developing systemic lupus erythematosus (SLE). In addition, there is an increased risk of lupus nephritis (2-fold), high-risk histological features, and resistance to treatment. This may account for the increased mortality rate compared to Caucasian patients, especially in women. In Phase One of the Accelerating Medicines Partnership (AMP) study, we used single-cell RNA sequencing on kidney biopsies from patients with active lupus nephritis to identify pathways that were differentially expressed in African-American patients.

Methods Single cell RNA sequencing was performed on renal biopsies obtained for clinical purpose for active nephritis using CEL-Seq2. Cell clusters with similar expression profile were identified using $\mathrm{t}$-distributed stochastic neighbor embedding $(\mathrm{t}-$ $\mathrm{SNE}$ ). First, the relative abundance of a cluster in AAs compared to Caucasian was determined using a logistic mixed model. Second, the differential expression profile was determined for each cell cluster and we applied Ingenuity Pathway Analysis (IPA) (QIAGEN Bioinformatics) to identify pathways of interest.

Results Samples from 13 AA and 7 Caucasian patients were obtained. Of the 3097 sequenced cell libraries, we used 2354 which passed our quality filter for a total of 30155 unique molecular identifiers. We identified 16 cell clusters including CD4, CD8, B and plasma cells, NK, myeloid cells, and tubular cells. We identified 2 cell clusters unique to African-American patients, a $\mathrm{T}$ and a $\mathrm{B}$ cell population with high expression of interferon inducible genes. We also identified that same cell populations may have differential gene expression profiles 